\title{
Multifunctional Braided Composite Rods for Civil Engineering Applications
}

\author{
Emilijia Zdraveva $^{1, a}$, Cristiana Gonilho-Pereira ${ }^{2, b}$, R. Fangueiro $^{1, c}$, \\ S. Lanceros-Mendez ${ }^{3, d}$, S. Jalali ${ }^{2, e}$, M. Araújo ${ }^{1, f}$
}

\section{Corresponding to; R. Fangueiro (rfang@det.uminho.pt)}

${ }^{1}$ Dept. of Textile Engineering, University of Minho, Campus de Azurém, Guimarães, Portugal

${ }^{2}$ Dept. of Civil Engineering, University of Minho, Campus de Azurém, Guimarães, Portugal,

${ }^{3}$ Dept. of Physics, University of Minho, Campus de Azurém, 4800-058 Guimarães, Portugal

aemilijia_zdraveva@net.hr, ${ }^{b}$ cristiana.pereira@civil.uminho.pt, 'rfang@det.uminho.pt, lanceros@fisica.uminho.pt, ${ }^{\mathrm{e}}$ said@civil.uminho.pt, ${ }^{f}$ maraujo@det.uminho.pt

Keywords: concrete, corrosion, braiding, composites, reinforcing, monitoring, electrical resistance, self-diagnosing structural materials.

Abstract. This paper presents the development of a braided reinforced composite rod (BCR) able to both reinforce and monitor the stress state of concrete elements. Carbon fibers have been used as sensing and reinforcing material along with glass fiber. Various composites rods have been produced using an author patented technique based on a modified conventional braiding machine. The materials investigated were prepared with different carbon fiber content as follows: $\mathrm{BCR}_{2}(77 \%$ glass $/ 23 \%$ carbon fiber), $\mathrm{BCR}_{3}$ (53\% glass $/ 47 \%$ carbon fiber), $\mathrm{BCR}_{4}(100 \%$ carbon fiber). BCRs have been tested under bending while the variation of the electrical resistance was simultaneously monitored. The correlations obtained between deformation and electrical resistance show the suitability of the rods to be used as sensors. The fractional resistance change versus strain plots show that the gage factor increases with decreasing carbon fiber content.

\section{Introduction}

The corrosion of steel reinforcing rebar is the main cause of concrete structures degradation. The most effective way to prevent concrete structures degradation is the steel replacement by a corrosion resistant reinforcing material, such as fiber-reinforced-polymer (FRP) composites. The types of FRP composites best suited for the reinforcement of concrete are those providing high strength, high stiffness, and environmental compatibility with concrete. Are rod-like elements, pultruded, shaped, and treated so that surface texture and undulations provide mechanical interlock with concrete.

Nevertheless, the interest in the safety of concrete structures has increased and monitoring damage of structures has become a main goal once is valuable for hazard mitigation. Therefore monitoring systems that can be applied to the reinforced concrete elements are required. The damage sensing is conventionally performed by attached or embedded damage sensors, such as optical fibers, acoustic sensors, among others, however these sensors have limited application because of high cost, low durability, and limited sensing volume and spatial resolution. One solution is that the materials themselves can possess a self-diagnosing function for fracture, the so called self-diagnosing structural materials - a multifunctional material $[1,2,3,4]$.

This research work is focused on the development of multifunctional material, a fiber-reinforced-polymer rod-like, based on braiding technology, for concrete elements reinforcement and monitoring, in order to overcome the main disadvantage of steel, e. g. corrosion. Self-diagnosing structural braided composite rods were produced, in a single step, with surface texture and undulations, reinforced with glass and carbon fiber, in different percentages. 


\section{Materials and Methods}

Three types of braided composite rods with different carbon fiber content $(23 \%, 77 \%$ and $100 \%)$ were prepared by the single step process of braiding and simultaneous resin impregnation. Braided reinforced composite rods have been produced on a vertical braiding machine with an incorporated impregnation system [8]. Table 1 shows the BCR composition. A total number of 14 samples were prepared and evaluated.

The testing procedure carried on the $\mathrm{BCR}$ is based on the electrical resistance measurement during simultaneous application of a deformation in a cyclic three-point bending test. The testing parameters are shown in Table 2.

Cyclic three-point bending tests (Table 3) were carried on a Universal Testing Machine Autograph IS (Shimadzu) 500N. The electrical resistance measurement was carried on a digital multi-meter (Agilent, 84401A). The electrical signal was acquired through golden wires attached to the cross section of the samples with silver paint.

Table 1 - BCR compositions

\begin{tabular}{ccccc}
\hline Type & Fiber composition & Fiber (\%) & $N^{o}$ of rovings & Linear mass (tex) \\
\hline $\mathrm{BCR}_{2}$ & E-glass/Carbon & $77 / 23$ & $18 / 3$ & $1600 / 900$ \\
\hline $\mathrm{BCR}_{3}$ & E-glass/Carbon & $53 / 47$ & $53 / 47$ & 900 \\
\hline $\mathrm{BCR}_{4}$ & Carbon & 100 & 12 & 900 \\
\hline
\end{tabular}

Table 2 - Dimensional characteristics of the BCR s

\begin{tabular}{lcc}
\hline BCR type & Diameter $(\mathrm{mm})$ & Linear mass $(\mathrm{g} / \mathrm{m})$ \\
\hline $\mathrm{BCR}_{2}($ glass $77 \%$, carbon $23 \%)$ & 5,66 & 36,16 \\
\hline $\mathrm{BCR}_{3}($ glass $53 \%$, carbon $47 \%)$ & 5,80 & 39,89 \\
\hline $\mathrm{BCR}_{4}($ carbon $100 \%)$ & 6,40 & 40,47 \\
\hline
\end{tabular}

Table 3 - Testing parameters

\begin{tabular}{cc}
\hline Parameter & Settings \\
\hline $\mathrm{N}^{\mathrm{o}}$ of cycles & 4 \\
\hline Load cell & $500 \mathrm{~N}$ \\
\hline Span & $6 \mathrm{~cm}$ \\
\hline Displacement limit & $0,5 \mathrm{~mm}$ \\
\hline Cross-head speed & $0,3 \mathrm{~mm} / \mathrm{min}$ \\
\hline Sampling interval & $500 \mathrm{~ms}$ \\
\hline
\end{tabular}

\section{Results and discussion}

In general, the electrical resistance during loading and unloading increases linearly at lower displacement values and nonlinearly at higher ones. In the case of the inverse response, during decrease of the electrical resistance and deformation increase, the nonlinearity is less evident. Whether it was a reverse or inverse response in both cases the tested sample showed the change of the electrical resistance in proper compliance with the change of its deformation.

The main factors influencing the type of response of each sample was the relative position of the fibers and the resin in the rods cross section, more precisely the carbon fiber position along the length of the rods. In this situation, increasing of the resistance with increasing deformation occurred when the carbon fibers are placed on the tensile side and the opposite behavior when their placement is on the compression side of the bending rod. This issue indicates the relevance of controlling carbon position on the rod cross-section. 
On the other hand, for each rod, the cycles are reproducible, confirming the reliable sensing property of the rods. The difference between the peak values in each of the cycles is around $0,01 \Omega$ to $0,02 \Omega$.

The range of the initial electrical resistance for the first typeBCR 2 (77\% glass, $23 \%$ carbon), vary from $1,46 \Omega$ to $3,92 \Omega$, for the second type $\mathrm{BCR}_{3}(53 \%$ glass, $47 \%$ carbon) from $1,11 \Omega$ to $1,50 \Omega$ and for the third $\mathrm{BCR}_{4}(100 \%$ carbon) from $0,80 \Omega$ to $1,37 \Omega$.

Furthermore, for the comparison of the sensing behavior differentiation due to carbon fiber content, the strain $\varepsilon\left({ }^{*} 10^{-2}\right)$ and the fractional resistance change $\Delta \mathrm{R} / \mathrm{R}_{0}$ of the three types of BCR are presented in Table 3. The strain is calculated from the displacement and the fractional resistance change is calculated from the electrical resistance change, the values from the two parameters are presented over time of 100, 300, 500 and 700 seconds.

Fig. 1 shows the correlation between the fractional resistance change $\left(\Delta R / R_{0}\right)$ and the flexural deformation ( $\varepsilon$ ) for of all the three rod types $\mathrm{BCR}_{2}\left(77 \%\right.$ glass, $23 \%$ carbon), $\mathrm{BCR}_{3}(53 \%$ glass, $47 \%$ carbon), $\mathrm{BCR}_{4}(100 \%$ carbon) in the time range $(0-100 \mathrm{sec} ; 200-300 \mathrm{sec} ; 400-500 \mathrm{sec} ; 600-700 \mathrm{sec})-$ first half of the cycle (loading sequence), or (100-200sec; 300-400sec; 500-600; 700-800sec) - second half of the cycle (unloading sequence).

Fig. $1 \mathrm{~A} 1$ and $1 \mathrm{~A} 2$ represents the positive type of response. Fig. $1 \mathrm{~B} 1$ and $1 \mathrm{~B} 2$ represent the negative type of response. The numbers 1 and 2, respectively, represent the loading and unloading sequence of the cycles. Furthermore the trend lines are presented for each of the plotted curves showing the linear accordance between the fractional resistance change and the deformation.
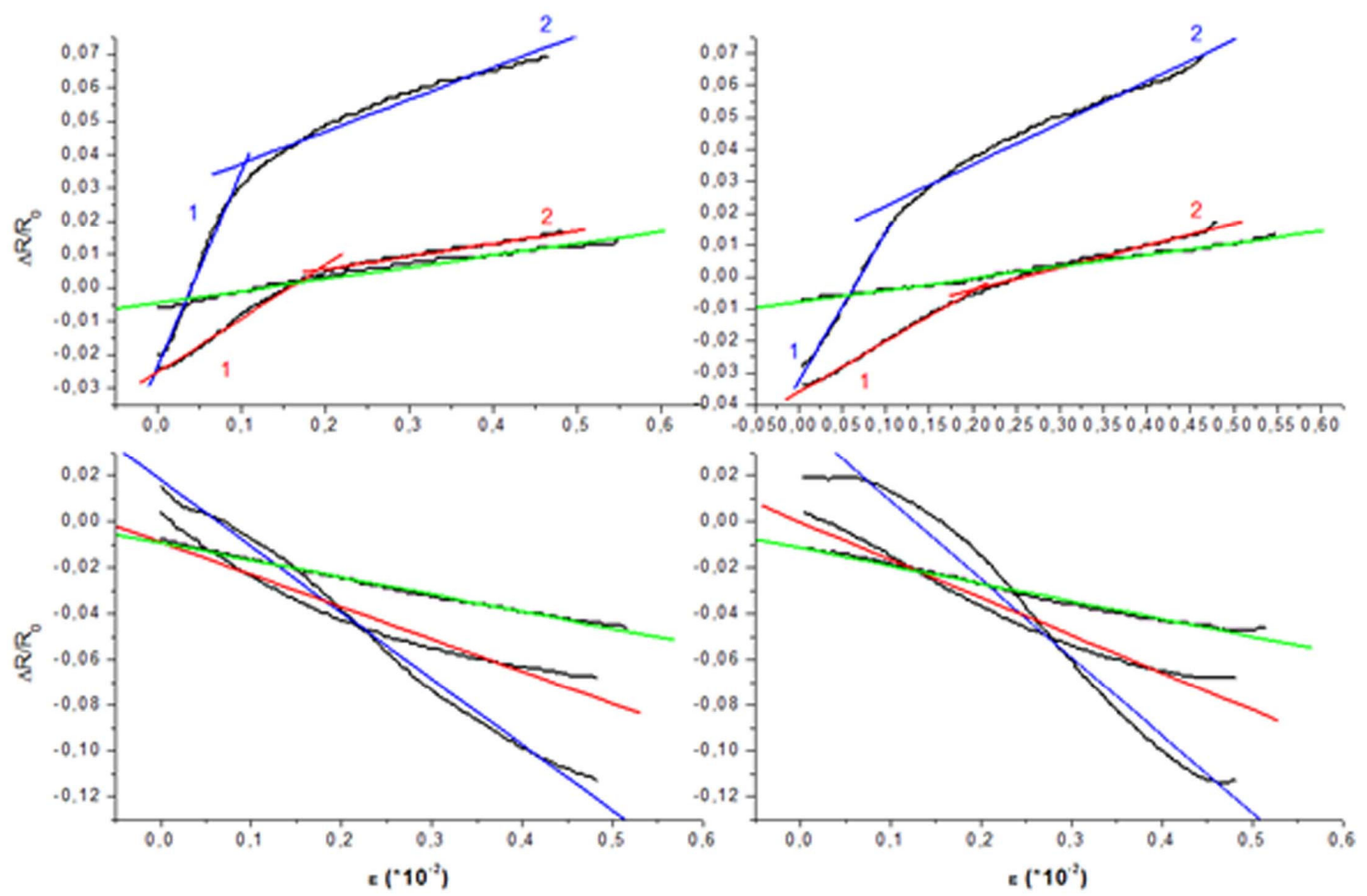

Fig. 1 - Fractional resistance change dependence on deformation. Comparison between the three types of tested samples: $\mathrm{BCR}_{2}, \mathrm{BCR}_{3}, \mathrm{BCR}_{4}$

1 - loading $\quad \mathrm{A}-$ positive response

2 - unloading $\quad \mathrm{B}$ - negative response

$\mathrm{BCR}_{2}(77 \%$ of glass, $23 \%$ of carbon $)$

$\mathrm{BCR}_{3}(53 \%$ of glass, $47 \%$ of carbon)

$\mathrm{BCR}_{4}(100 \%$ of carbon $)$ 
As can be seen in Table 5, the gage factor for type $\mathrm{BCR}_{2}(77 \%$ glass, $23 \%$ carbon $)$ is almost five times higher than that for the type $\mathrm{BCR}_{3}(53 \%$ glass, $47 \%$ carbon $)$ and $\mathrm{BCR}_{4}(100 \%$ carbon $)$. The gage factor of type $\mathrm{BCR}_{3}(53 \%$ glass, $47 \%$ carbon) is slightly higher.

Table 5 - Gage factors (GF) and squared regression values $\left(\mathrm{R}^{2}\right)$ of established trend equations

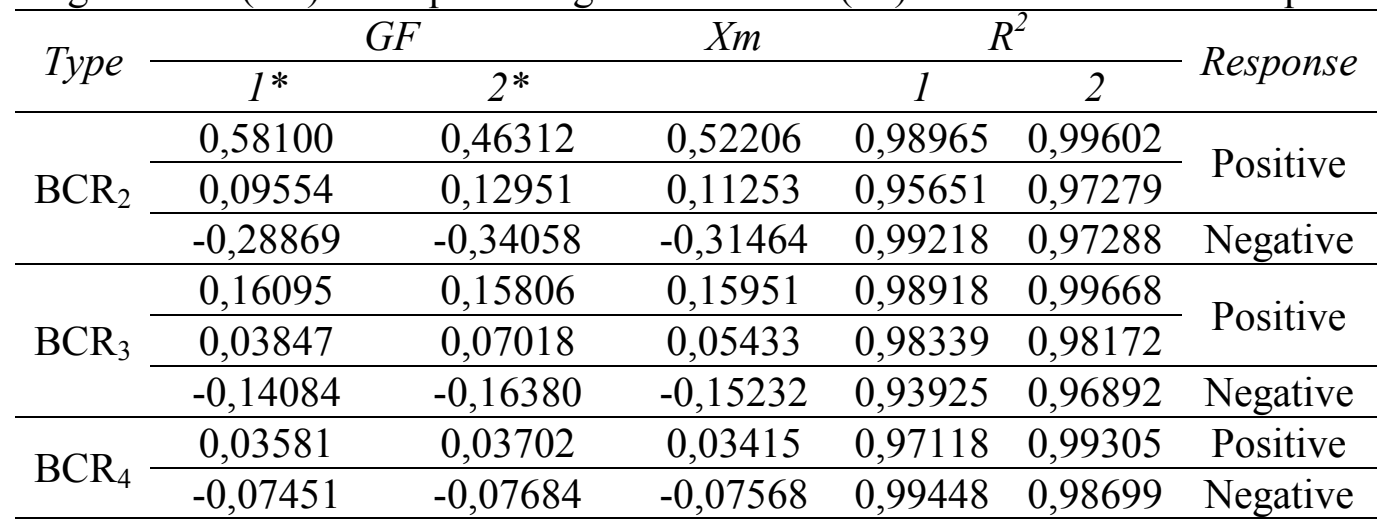

The gage factor, known as the strain-sensing factor, shows the sensing behavior of the BCR samples. It increases with the decreasing of the carbon fiber percentage. This means that type $\mathrm{BCR}_{2}(77 \%$ glass, $23 \%$ carbon) has the most reliable monitoring behavior.

The gage factors calculated for the BCRs are all less than 1 due to the higher percentage of the carbon component [4]. On the other hand, the main advantage of the present materials is the superior reinforcing capabilities and therefore the combination of reinforcing and sensing capabilities.

\section{Conclusions}

This work represents a contribution to develop monitoring systems for civil engineering structures in order to improve their sustainability. The final goal of this study is to develop such on-line monitoring system, by the application of braided reinforced composite rods (BCR) in concrete structures.

This paper reported on the influence of carbon fiber amount in the sensing proformance of braided reinforced rods using glass and carbon. It was proven that all three types of BCR used can stand as a self-sensing material. The electrical contact set-up was effective in the purpose of resistance stabilization and measurent.Two types of responces were obtained by the BCR. Positive GF, in the case of the carbon fibreplaced inthe area subjected to tensile and negative GF, in the case of the carbon fibre placed in the compressive side of the rod. Furthermore, the GF increased with decreasing carbon fiber content. The most reliable monitoring behavior was given by type $\mathrm{BCR}_{2}(77 \%$ glass, $23 \%$ carbon) with the smallest carbon fiber content.

An optimum balance between the carbon and the glass fiber contents needs to be established in order to provide both rod functions, i.e., the monitoring and the reinforcing one.

\section{References}

[1] D. D. L. Chung, Self-monitoring structural materials. Composite Materials Research Laboratory, State University of New York at Buffalo, Buffalo, NY 14260-4400, USA, 1997.

[2] Yoshiki Okuhara, Hideaki Matsubara, Memorizing maximum strain in carbon - fibre reinforced plastic composites by measuring electrical resistance under pre - tensile stress. Comp Sci and Tech 65 (2005) 2148-2155.

[3] F. Nanni, F. Auricchio, F. Sarchi, G. Forte, G. Gusmano, Self - sensing CF-GFRP rods as mechanical reinforcement and sensors of concrete beams. Smart Mater Struct 15 (2006) 182-186.

[4] Inpil Kang, et al; Introduction to carbon nanotube and nanofiber smart materials. Composites: part B 37, March 2006, 382-394. 
Multi-Functional Materials and Structures III

doi:10.4028/www.scientific.net/AMR.123-125

Multifunctional Braided Composite Rods for Civil Engineering Applications doi:10.4028/www.scientific.net/AMR.123-125.149 